Journal of Dynamics and Differential Equations, Vol. 6, No. 4, 1994

\title{
Uniform Persistence and Flows Near a Closed Positively Invariant Set
}

\author{
H. I. Freedman, ${ }^{1}$ Shigui Ruan, ${ }^{2}$ and Moxun Tang ${ }^{3}$
}

Received December 16, 1992; revised October 5, 1993

\begin{abstract}
In this paper, the behavior of a continuous flow in the vicinity of a closed positively invariant subset in a metric space is investigated. The main theorem in this part in some sense generalizes previous results concerning classification of the flow near a compact invariant set in a locally compact metric space which was described by Ura-Kimura (1960) and Bhatia (1969). By applying the obtained main theorem, we are able to prove two persistence theorems. In the first one, several equivalent statements are established, which unify and generalize earlier results based on Liapunov-like functions and those about the equivalence of weak uniform persistence and uniform persistence. The second theorem generalizes the classical uniform persistence theorems based on analysis of the flow on the boundary by relaxing point dissipativity and invariance of the boundary. Several examples are given which show that our theorems will apply to a wider varity of ecological models.
\end{abstract}

KEY WORDS: Positively invariant set; limit set; prolongational set; dissipativeness; persistence.

AMS SUBJECT CLASSIFICATIONS: $34 \mathrm{C} 35,58 \mathrm{~F} 25,92 \mathrm{~A} 15$.

\section{INTRODUCTION}

Since Butler et al. (1986) and Butler and Waltman (1986) published two consecutive papers in persistence theory, a number of papers on persistence in dynamical systems have appeared; see, for example, Dunbar et al. (1986), Fonda (1988), Garay (1989), Hofbauer (1989), Hofbauer and So

\footnotetext{
${ }^{1}$ Applied Mathematics Institute, Department of Mathematics, University of Alberta, Edmonton, Alberta, Canada T6G 2G1.

${ }^{2}$ Department of Mathematics and Statistics, McMaster University, Hamilton, Ontario, Canada L8S 4K1. Current address: Department of Mathematics, Statistics and Computer Science, Dalhousie University, Halifax, Nova Scotia, Canada B3H $3 J 5$.

${ }^{3}$ Department of Mathematics, University of Alberta, Edmonton, Alberta, Canada T6G 2G1.
} 
(1989), Hale and Waltman (1989), Freedman and So (1989), Freedman and Moson (1990), Tang (1990), Teng and Duan (1990), Yang and Ruan (1992), and Thieme (1993). There are also two recent survey papers by Hutson and Schmitt (1992) and Waltman (1992) on persistence theory as it stands now.

As far as we understand the literature, there are basically two distinct techniques utilized in determining persistence criteria:

(1) analyzing the flow on the boundary and

(2) using Liapunov-like persistence functions.

The first approach was proposed by Freedman and Waltman (1984) to study persistence in three interacting predator-prey populations. To use this approach one needs the so-called Butler-McGehee lemma, which says that if a trajectory, not on the stable manifold of a given isolated hyperbolic equilibrium $P$, has that equilibrium in its omega limit set, then its omega limit set also contains points on the stable and unstable manifolds of the equilibrium different from $P$. This lemma has been extended to a compact isolated invariant set and a continuous flow on a locally compact metric space by Butler and Waltman (1986), to a continuous semiflow by Dunbar et al. (1986), and to a complete metric space (not necessarily locally compact) by Hale and Waltman (1989). Recently, it also has been generalized by Yang and Ruan (1992) in such a way as to encompass orbits from a set rather than a single point and to consider the closure of the union of the omega limit sets of all points in that set. With such a generalization, a uniform persistence theorem has been established for certain dynamical systems which are not necessarily dissipative. Garay (1989) generalized the main theorem of Butler and Waltman (1986) by using Conley's (1978) theory of invariant sets and a theorem obtained by Ura and Kimura (1960) and Bhatia (1969). Briefly speaking, the UraKimur-Bhatia theorem means that in a local compact space, either an isolated compact invariant set is asymptotically stable (positively or negatively) or there exist two points not in the compact set, whose omega or alpha limit sets belong to the compact set, respectively.

The approach of using Liapunov-like functions has appeared in various forms, for example, Gard and Hallam (1979), Hofbauer (1980), Hutson (1984), Gard (1987), Fonda (1988), Hofbauer and So (1989), Fernandes and Zanolin (1989), and Freedman and Ruan (1994). The nicest statement is due to Fonda (1988), who stated the result in terms of repellers. This result has been generalized (and reproved) by Hofbauer and So (1989) to discrete semidynamical systems. For more details and more references about the two approaches and their applications in biological 
models, we refer to the survey papers by Hutson and Schmitt (1992) and Waltman (1992).

In this paper, we first investigate the behavior of the flow in a metric space (not necessarily locally compact) near a closed positively invariant set (not necessarily compact); hence, our results generalize the theorem obtained by Ura and Kimura (1960) and Bhatia (1969) and the ButlerMcGehee lemma (Butler and Waltman, 1986). The generalization allows us to prove two persistence theorems. In the first theorem, several equivalent statements are established, which include the statement that if the flow is dissipative on a subset of the interior of the positive cone, weak uniform persistence is equivalent to uniform persistence and is also equivalent to those uniform persistence conditions obtained by using Liapunov-like functions. The first equivalence improves a similar equivalence obtained by Freedman and Moson (1990). Recently, Thieme (1993) has obtained some very interesting results about this kind of equivalence. Some earlier results are unified and generalized in the first theorem.

In the second theorem, we generalize the classical theorem of Butler and Waltman (1986) by relaxing point dissipativity and invariance of the boundary of the stated subset. The flow is required to be point dissipative only on a subset of the interior of the positive cone near the boundary. Similar attempts have been made by Teng and Duan (1990), Thieme (1993), and Yang and Ruan (1992).

The rest of this paper is organized as follows. In Section 2, basic notations and definitions are introduced. The behavior of the flow near a closed positively invariant set is discussed in Section 3. In Section 4, two unified and generalized persistence theorems are given. In the last section, we give several examples to illustrate our results.

\section{DEFINITIONS AND NOTATIONS}

In this section, we give some basic notations and definitions on dynamical systems which we require for this paper.

Let $X$ be a metric space with metric $d$. Let $R$ denote the set of real numbers with the usual topological and algebraic structure, and let $R^{+}$and $R^{-}$denote the sets of nonnegative and nonpositive real numbers, respectively. We consider a continuous flow $\mathscr{F}=(X, R, \pi)$ defined on $X$, where $\pi: X \times R \rightarrow X$ is a continuous map such that $\pi(x, 0)=x$ for all $x \in X$ and $\pi(\pi(x, t), s)=\pi(x, t+s)$ for all $x \in X, t, s \in R$. If $M \subset X$ and $K \subset R$, then $\pi(M, K)$ denotes the set

$$
\{\pi(x, t): x \in M, t \in K\}
$$


For any $M \subset X$, we define

$$
\gamma(M)=\pi(M, R), \quad \gamma^{+}(M)=\pi\left(M, R^{+}\right), \quad \gamma^{-}(M)=\pi\left(M, R^{-}\right)
$$

For singleton $M=\{x\}$, the corresponding sets are denoted $\gamma(x), \gamma^{+}(x)$, $\gamma^{-}(x)$ and are, respectively, called the trajectory, positive trajectory, and negative trajectory of $x$. A set $M \subset X$ is called invariant, positively invariant, or negatively invariant if and only if $M=\gamma(M), M=\gamma^{+}(M)$ or $M=\gamma^{-}(M)$, respectively. The boundary, closure, and interior of a set $M \subset X$ are denoted $\partial M, \bar{M}$, and $\dot{M}$, respectively.

For any $\varepsilon>0$ and $M \subset X$, we define

$$
\begin{aligned}
S(M, \varepsilon) & =\{x: x \in X, d(x, M)<\varepsilon\} \\
S[M, \varepsilon] & =\{x: x \in X, d(x, M) \leqslant \varepsilon\} \\
H(M, \varepsilon) & =\{x: x \in X, d(x, M)=\varepsilon\}
\end{aligned}
$$

The limit sets, prolongational sets, and prolongational limit sets of a point $x \in X$ are defined as follows.

Definition 2.1. For each $x \in X$, the set

$$
\begin{array}{r}
D^{+}(x)=\left\{y \in X: \text { there are sequences }\left\{x_{n}\right\} \subset X,\left\{t_{n}\right\} \subset R^{+}\right. \\
\text {such that } \left.x_{n} \rightarrow x, \pi\left(x_{n}, t_{n}\right) \rightarrow y \text { as } n \rightarrow \infty\right\}
\end{array}
$$

is called the first positive prolongation of $x$. The first negative prolongation $D^{-}(x)$ is defined by requiring $\left\{t_{n}\right\} \subset R^{-}$. The first prolongational limit set of $x \in X$ is defined as

$$
\begin{aligned}
& J^{+}(x)=\left\{y \in X: \text { there are sequences }\left\{x_{n}\right\} \subset X,\left\{t_{n}\right\} \subset R^{+}\right. \\
& \text {such that } \left.x_{n} \rightarrow x, t_{n} \rightarrow \infty, \pi\left(x_{n}, t_{n}\right) \rightarrow y \text { as } n \rightarrow \infty\right\}
\end{aligned}
$$

The first negative prolongational limit set $J^{-}(x)$ is defined by requiring $\left\{t_{n}\right\} \subset R^{-}$and $t_{n} \rightarrow-\infty$ as $n \rightarrow \infty$. The positive (or omega) limit set of $x \in X$ is defined to be

$$
\begin{array}{r}
A^{+}(x)=\left\{y \in X: \text { there is a sequence }\left\{t_{n}\right\} \subset R^{+}\right. \text {with } \\
\left.t_{n} \rightarrow+\infty \text { and } \pi\left(x, t_{n}\right) \rightarrow y \text { as } n \rightarrow \infty\right\}
\end{array}
$$

The negative (or alpha) limit set $\Lambda^{-}(x)$ is defined by requiring $\left\{t_{n}\right\} \subset R^{-}$ and $t_{n} \rightarrow-\infty$ as $n \rightarrow \infty$.

Obviously, for any $x \in X$, we have $D^{+}(x) \supset \gamma^{+}(x), D^{-}(x) \supset \gamma^{-}(x)$, $J^{+}(x) \supset A^{+}(x)$, and $J^{-}(x) \supset \Lambda^{-}(x)$. 
Proposition 2.2. For any $x \in X, D^{+}(x)$ is closed positively invariant, $\Lambda^{+}(x)$ and $J^{+}(x)$ are closed invariant. Furthermore,

$$
D^{+}(x)=\gamma^{+}(x) \cup J^{+}(x)
$$

Similar results hold for $\mathrm{D}^{-}(x),{A^{-}}^{-}(x)$, and $J^{-}(x)$.

For the above definitions and results, we refer to Bhatia and Szegö (1970) and Bhatia (1970).

Definition 2.3. For any $x \in X$, if $\overline{\gamma^{+}(x)}$ is compact, then the flow $\mathscr{F}$ is said to be quasi-dissipative at $x$. Let $M \subset X$ be a nonempty set. If $\mathscr{F}$ is quasi-dissipative at each point $x \in M$, then $\mathscr{F}$ is said to be quasi-dissipative over $M$.

If a flow $\mathscr{F}$ is quasi-dissipative over $M$, then for any point $x \in M$, the omega limit set $\Lambda^{+}(x)$ is nonempty, compact, connected, and invariant. Note that the definition of quasi-dissipativity coincides with the definition of positive compactness in Sell (1967).

Definition 2.4. For any $x \in X$, if there exists a compact neighborhood $U$ of $x$, and a compact set $V$ such that for any $y \in U$, there is some time $t(y)>0$ such that $\pi(y, t) \in V^{\circ}$ for all $t \geqslant t(y)$, then the flow $\mathscr{F}$ is said to be locally dissipative at $x$. The flow $\mathscr{F}$ is locally dissipative over a nonempty set $M \subset X$ if $\mathscr{F}$ is locally dissipative at each point $x \in M$.

By Definition 2.4 , if $\mathscr{F}$ is locally dissipative at $x$ with corresponding sets $U$ and $V$, then one can find $t(U)>0$ such that for all $y \in U$ and $t \geqslant t(U), \pi(y, t) \in V^{\circ}$ holds. The set $\pi\left(U, R^{+}\right)$is relatively compact.

Definition 2.5. The flow $F$ is point dissipative over a nonempty set $M \subset X$ if there exists a compact set $N \subset X$ such that for any $y \in M$, there exists $t(y)>0$ such that for any $t \geqslant t(y), \pi(y, t) \in N^{2}$.

For the above definitions and their inter relations, we refer to Hale (1988) and Teng and Duan (1990). If the space $X$ is locally compact, the above definition of point dissipativity coincides with dissipativity given by Butler et al. (1986) and Butler and Waltman (1986).

Definition 2.6. A nonempty subset $M \subset X$ is called an isolated set if there exists $\varepsilon>0$ such that for any invariant set $N$ contained entirely in $S[M, \varepsilon]$, we have $N \subset M$. 
If the set $M$ is closed invariant, our definition coincides with the one given by Butler et al. (1986). Note that in Definition 2.6, it is not required that there exists an invariant set contained in $M$. For example, according to Definition 2.6, any regular point in Euclidean space $R^{n}$ is isolated.

\section{FLOWS NEAR A CLOSED POSITIVELY INVARIANT SET}

Let $X$ be a metric space with metric $d$, and $\mathscr{F}$ be a continuous flow defined on $X$. In this section, we discuss the behavior of the flow near a closed positively invariant set in the metric space $X$. The main result of this section is Theorem 3.7, which gives a classification of possible behavior of the flow near a closed positively invariant set $E$ in the metric space $X$ where the flow $\mathscr{F}$ is assumed to be point dissipative on $S[E, \alpha] / E$, the set of all points belonging to $S[E, \alpha]$ but not $E$, and $\alpha$ is a suitable positive constant.

In the case that $X$ is locally compact and $E$ is compact invariant subset of $X$, a classification of behavior of the flow in the vicinity of $E$, described by Ura and Kimura (1960) and Bhatia (1969), may be stated as follows.

Ura-Kimura-Bhatia Theorem. Let the metric space $X$ be locally compact and $E \subset X$ be a compact invariant subset of $X$. Then one of the following statements holds.

(i) The set $E$ is not isolated, that is, for any neighborhood $\mathscr{U}$ of $E$, there exists an $x \notin E$ with $\gamma(x) \subset \mathscr{U}$. (Note that since $E$ is invariant, $\gamma(x) \cap E=\varnothing)$.

(ii) There exist $y \notin E$ and $z \notin E$ such that $\varnothing \neq \Lambda^{+}(y) \subset E$ and $\varnothing \neq \Lambda^{-}(z) \subset E$.

(iii) $E$ is positively (negatively) asymptotically stable, that is, any neighborhood $\mathscr{U}$ of $E$ contains a positively (negatively) invariant neighborhood $\mathcal{Q}$ of $E$ such that for any $x \in \mathscr{U}, \varnothing \neq \Lambda^{+}(x) \subset E$ (or $\varnothing \neq \Lambda^{-}(x) \subset E$, respectively).

In Theorem 3.7, the space $X$ is not required to be locally compact and the set $E$ is required only to be closed and positively invariant. It is in this sense that the above theorem is generalized.

If the subset $E$ is reduced to an equilibrium of the flow $F$, and if we make the further assumption that $E$ is an isolated hyperbolic equilibrium, then from the above theorem we can see that either $E$ is asymptotically stable (positively or negatively) or there exist two points $y$ and $z$ different from $E$ such that $\Lambda^{+}(y)=E$ and $\Lambda^{-}(z)=E$. In fact, we have the following 
so-called Butler-McGehee lemma (Freedman and Waltman, 1984; Butler and Waltman, 1986), which has a stronger conclusion in this case.

Butler-McGehee Lemma. Let $E$ be an isolated hyperbolic equilibrium in a locally compact metric space $X$, and suppose there exists $x \neq E$ such that $E \in A^{+}(x)$ but $E \neq \Lambda^{+}(x)$. Then there exist points $y$ and $z$ in $\Lambda^{+}(x)$ different from $E$ such that $A^{+}(y)=E$ and $\Lambda^{-}(z)=E$.

Our first result, Theorem 3.1 of this section, may be regarded as a generalization of the Butler-McGehee lemma in some sense. We mention here that Sell and Sibuya (1967) described a classification for the behavior of solutions of the nonautonomous differential equations in the vicinity of a critical point under some general assumptions and their result is similar to the above Ura-Kimura-Bhatia theorem if the compact set $E$ is reduced to an equilibrium and the system is reduced to the autonomous case.

Theorem 3.1. Let $E$ be a closed positively invariant subset of $X$ and $X$ be a point in $X$ with $d(x, E)>0$. Suppose the flow $\mathscr{F}$ is locally dissipative at $x$ and $D^{+}(x) \cap E \neq \varnothing$. Then for any $0<\varepsilon<d(x, E)$, there exists $y \in H(E, \varepsilon)$ such that $\Lambda^{+}(y) \subset S[E, \varepsilon]$.

Proof. Take $z \in D^{+}(x) \cap E$. Then there exist sequences $\left\{x_{n}\right\} \subset X$ and $\left\{t_{n}\right\} \subset R^{+}$such that $x_{n} \rightarrow x, \pi\left(x_{n}, t_{n}\right) \rightarrow z$ as $n \rightarrow \infty$. Since $\mathscr{F}$ is locally dissipative at $x$, we can choose a closed neighborhood $U_{x}$ of $x$ such that $U_{x} \cap S[E, \varepsilon]=\varnothing$ and $\pi\left(U_{x}, R^{+}\right) \subset V_{x}$, where $V_{x}$ is a compact set. Also,

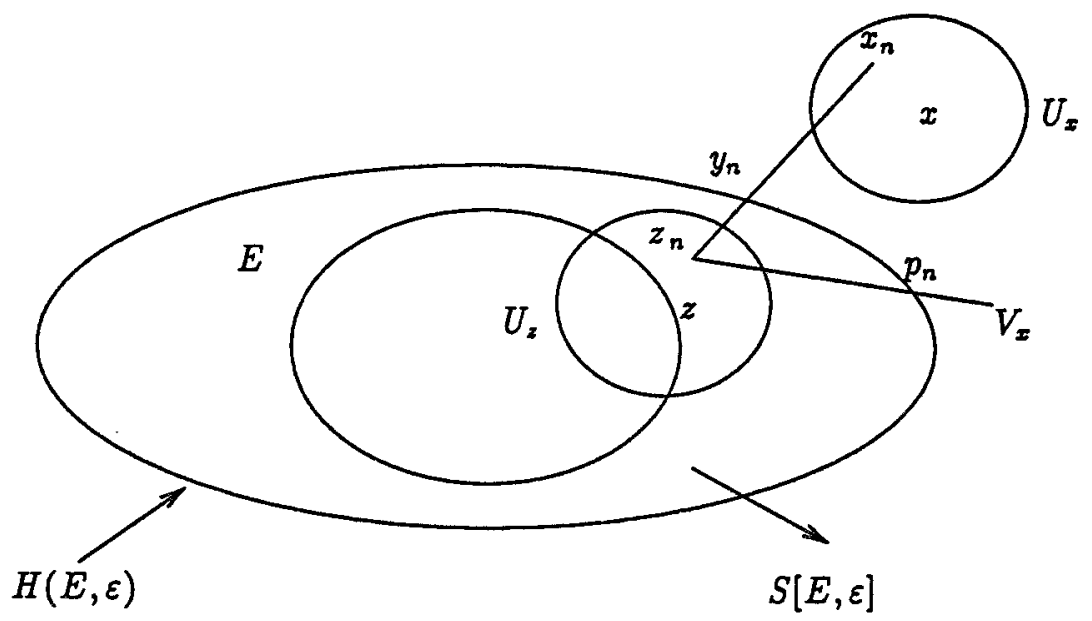

Fig. 1. 
we can choose a closed neighborhood $U_{z}$ of $z$ such that $U_{z} \subset S[E, \varepsilon / 2]$, where $0<\varepsilon<d(x, E)$ (Fig. 1).

Without loss of generality, we assume that $\left\{x_{n}\right\} \subset U_{x}$ and $\left\{\pi\left(x_{n}, t_{n}\right)\right\} \subset U_{z}$. Let $z_{n}=\pi\left(x_{n}, t_{n}\right)$. Then $\left\{z_{n}\right\} \subset U_{z}$. The positive trajectories $\gamma^{+}\left(x_{n}\right)$ must touch the set $H(E, \varepsilon)$ sometime between $t=0$ and $t_{n}$. Define

$$
\tau_{n}=\inf \left\{t: 0<t<t_{n}, \pi\left(x_{n}, t\right) \in H(E, \varepsilon), \pi\left(x_{n},\left(t, t_{n}\right)\right) \in S(E, \varepsilon)\right\}
$$

Clearly $0<\tau_{n}<t_{n}$. Let $y_{n}=\pi\left(x_{n}, \tau_{n}\right)$, then $z_{n}=\pi\left(y_{n}, t_{n}-\tau_{n}\right), y_{n} \in H(E, \varepsilon)$. If there exists some $y_{n} \in H(E, \varepsilon)$ such that for all $t \in R^{+}, \pi\left(y_{n}, t\right) \in S[E, \varepsilon]$, then let $y=y_{n}$. Then $A^{+}(y) \subset S[E, \varepsilon]$ and we are done. So in the following we suppose that for every point $x_{n}$, there is $s_{n}>t_{n}$ satisfying

$$
s_{n}=\max \left\{t: t_{n}<t<+\infty, \pi\left(x_{n}, t\right) \in H(E, \varepsilon), \pi\left(x_{n},\left(t_{n}, t\right)\right) \in S(E, \varepsilon)\right\}
$$

We denote $p_{n}=\pi\left(x_{n}, s_{n}\right)$. Then $p_{n} \in H(E, \varepsilon), 0<\tau_{n}<t_{n}<s_{n}<+\infty$. Note that $\left\{y_{n}\right\} \subset V_{x} \cap H(E, \varepsilon),\left\{p_{n}\right\} \subset V_{x} \cap H(E, \varepsilon)$ and since $V_{x} \cap H(E, \varepsilon)$ is compact, we can choose a convergent subsequence of $\left\{y_{n}\right\}$ and a convergent subsequence of $\left\{p_{n}\right\}$ which we also rewrite as $\left\{y_{n}\right\}$ and $\left\{p_{n}\right\}$. Then there exists $y \in H(E, \varepsilon)$ and $p \in H(E, \varepsilon)$ such that

$$
\lim _{n \rightarrow \infty} y_{n}=y, \quad \lim _{n \rightarrow \infty} p_{n}=p
$$

Now we prove that $s_{n}-\tau_{n} \rightarrow \infty$ as $n \rightarrow \infty$. Actually at this time we can prove that $s_{n}-t_{n} \rightarrow \infty$ as $n \rightarrow \infty$. If this is not true, we could find a sequence of the form $\left\{s_{n}-t_{n}\right\}$ and a constant $T>0$ and, without loss of generality, say $s_{n}-t_{n} \rightarrow T \geqslant 0$ as $n \rightarrow \infty$. Then we have that

$$
p=\lim _{n \rightarrow \infty} \pi\left(p_{n}, s_{n}\right)=\lim _{n \rightarrow \infty} \pi\left(z_{n}, s_{n}-t_{n}\right)=\pi(z, T)
$$

This is impossible since $z \in E, p \notin E$, and $E$ is positively invariant. Hence for any $t>0$, there exists an $N_{t}>0$ such that for any $n>N_{t}$, we have $\pi\left(y_{n}, t\right) \in S[E, \varepsilon]$, and since $\pi(y, t)=\lim _{n \rightarrow \infty} \pi\left(y_{n}, t\right)$, then $\pi(y, t) \epsilon$ $S[E, \varepsilon]$. Therefore $\gamma^{+}(y) \subset S[E, \varepsilon]$ and then $A^{+}(y) \subset S[E, \varepsilon]$. This completes the proof.

Remark 1. In the case that every positive trajectory $\gamma^{+}\left(x_{n}\right)$ exits the set $S[E, \varepsilon]$ at the point $p_{n}$, one can see that for the limit point $p$ of $\left\{p_{n}\right\}$, we have $A^{-}(p) \subset S[E, \varepsilon]$ and $y \in D^{+}(x), p \in J^{+}(x)$.

Remark 2. If the set $E$ is isolated with $\alpha>0$, i.e., every invariant set $K$ contained entirely in $S[E, \alpha]$ satisfies $K \subset E$, in addition to the assump- 
tion of Theorem 3.1, then for any $0<\varepsilon<\min \{\alpha, d(x, E)\}$, one can find a point $y \in H(E, \varepsilon)$ such that $A^{+}(y) \subset E$.

Remark 3. If the set $E$ is compact invariant and the metric space $X$ is locally compact, then the assumption of local dissipativeness at $x$ can be removed for sufficiently small $\varepsilon>0$.

If one requires that the set $X / E$ is a positively invariant subset instead of $E$ being positively invariant, where $E$ is a nonempty closed set, then the conclusion of Theorem 3.1 also holds. In fact, we have the following result.

Theorem 3.2. Let $E$ be a closed subset of $X$ such that the flow $\mathscr{F}$ is positively invariant over $X / E$. Let $x \notin E$ be such that $d(x, E)>0$, and let the flow $\mathscr{F}$ be locally dissipative at $x, D^{+}(x) \cap E \neq \varnothing$. Then for any $0<\varepsilon<$ $d(x, E)$, there exists $y \in H(E, \varepsilon)$ such that $A^{+}(y) \subset S(E, \varepsilon)$.

Proof. The proof is similar to that of Theorem 3.1. In this case, after constructing sequences $\left\{\tau_{n}\right\},\left\{t_{n}\right\}$, and $\left\{s_{n}\right\}$ similar to those constructed in the proof of Theorem 3.1, one can show that $t_{n}-\tau_{n} \rightarrow \infty$.

If $E$ is a closed positively invariant subset of $X$ with nonempty boundary $\partial E$ and nonempty interior $E^{\circ}$, then $E^{\circ}$ is also positively invariant, but $\partial E$ is in general not positively invariant. As in Theorem 3.2, we have the following theorem.

Theorem 3.3. Let $E$ be a closed, positively invariant subset of $X$ with nonempty $E^{\prime}$ and $\partial E$. Let $x \in \mathcal{E}$ and the flow $\mathscr{F}$ be locally dissipative at $x$. If $D^{+}(x) \cap \partial E \neq \varnothing$, then for any $0<\varepsilon<d(x, \partial E)$, there exists $y \in H(\partial E, \varepsilon)$ such that $\Lambda^{+}(y) \subset S[\partial E, \varepsilon]$.

Note that for any $x \in X, A^{+}(x) \subset J^{+}(x) \subset D^{+}(x)$. Hence the following corollaries are valid.

Corollary 3.4. The conclusions of Theorems 3.1, 3.2, and 3.3 hold if the set $D^{+}(x)$ is replaced by $J^{+}(x)$.

Corollary 3.5. The conclusions of Theorems 3.1, 3.2, and 3.3 hold if the set $D^{+}(x)$ is replaced by $A^{+}(x)$. In this case, the flow $F$ is required only to be quasi-dissipative at $x$ instead of locally dissipative at $x$.

The proof of Corollary 3.5 is similar to that of Theorem 3.1. The only difference is that every point in $\left\{x_{n}\right\}$ is $x$ and the compact set $V_{x}$ should be changed to $\overline{\gamma^{+}(x)}$.

By Theorem 3.1 and Remarks 1 and 2, we also have the following. 
Corollary 3.6. Suppose $E$ is a closed, positively invariant subset of $X$, $x \notin E$, and $\gamma^{+}(x)$ is compact. If $\Lambda^{+}(x) \cap E \neq \varnothing, x_{0} \in A^{+}(x) / E$, and the set $E$ is isolated with a corresponding number $\alpha>0$ such that any invariant set $K$ contained entirely in $S[E, \alpha]$ satisfies $K \subset E$, then for any $0<\varepsilon<$ $\min \left\{\alpha, d\left(x_{0}, E\right)\right\}$, there exist points $y \in H(E, \varepsilon) \cap A^{+}(x)$, and $p \in H(E, \varepsilon) \cap$ $A^{+}(x)$ such that

$$
\Lambda^{+}(y) \subset E, \quad A^{-}(p) \subset E
$$

Remark 4. Corollary 3.6 is similar to Theorem 2.2 of Dunbar et al. (1986), in which the map $\pi$ is a local semiflow. Also by Remark 3, Corollary 3.6 can be reduced to Theorem 4.1 of Butler and Waltman (1986).

Theorem 3.7. Let $E$ be a closed positively invariant set for a continuous flow $\mathscr{F}$ on a metric space $X$. Suppose there exists $\alpha>0$ such that $\mathscr{F}$ is point dissipative on $S[E, \alpha] / E$, then one of the following statements holds.

(i) The set $E$ is not isolated, that is, for any $\varepsilon>0$, there exists an invariant set $K \subset S[E, \varepsilon]$ and $K \not \subset E$.

(ii) There exists $y \in S(E, \alpha) / E$, such that $A^{+}(y) \subset E$.

(iii) There is $\varepsilon>0$ such that for any $x \in S[E, \alpha] / E, \lim _{t \rightarrow \infty} d(\pi(x, t)$, $E) \geqslant \varepsilon$.

Proof. Without loss of generality, we assume that (i) and (ii) do not hold and then show that (iii) holds.

Choose $0<\delta<\alpha$, such that the neighborhood $S[E, \delta]$ of $E$ is isolated, that is, if $K$ is an invariant set and $K \subset S[E, \delta]$, then $K \subset E$.

If there exists $x \in S[E, \alpha]$ such that $\Lambda^{+}(x) \cap E \neq \varnothing$, take $0<\varepsilon_{0}<$ $\min \{d(x, E), \delta\}$. From Corollary 3.5 and Remark 2 , there exists $y \in H\left(E, \varepsilon_{0}\right)$ such that $A^{+}(y) \subset E$, which is impossible since $y \in S[E, \alpha] / E$ and we assume that (ii) does not hold. Hence for any $x \in S[E, \alpha] / E, \Lambda^{+}(x) \cap E=\varnothing$. Moreover, for any $x \notin E, A^{+}(x) \cap E=\varnothing$.

Since the flow $\mathscr{F}$ is point dissipative over $S[E, \alpha] / E$, we can find a compact set $N$ such that for any $y \in S[E, \alpha] / E$, there exists $T_{y}>0$ such that $\pi(y, t) \in N$ when $t>T_{y}$. Define

$$
\Omega=\left\{\Lambda^{+}(y): y \in S[E, \alpha] / E\right\}
$$

Then $\Omega \subset N$ and $\bar{\Omega} \subset N$.

Choose a sequence $\left\{\varepsilon_{n}\right\}, 0<\varepsilon_{n}<\delta$ such that $\lim _{n \rightarrow \infty} \varepsilon_{n}=0$. If (iii) does not hold, then for any $\varepsilon_{n}$, we can find $x_{n} \in S[E, \alpha] / E$ such that $\Lambda^{+}\left(x_{n}\right) \cap H\left(E, \varepsilon_{n}\right) \neq \varnothing$. In this case, we must have $A^{+}\left(x_{n}\right) \cap H(E, \delta) \neq \varnothing$. 
Otherwise $A^{+}\left(x_{n}\right) \subset S[E, \delta]$ and then $\Lambda^{+}\left(x_{n}\right) \subset E$, which is impossible, and

$$
\inf \left\{d(y, E), y \in \Lambda^{+}\left(x_{n}\right)\right\}<\varepsilon_{n}, \quad \sup \left\{d(y, E), y \in \Lambda^{+}\left(x_{n}\right)\right\}>\delta
$$

Choose sufficiently large $\tau_{n}>0, t_{n}>0$ with $t_{n}-\tau_{n}>0$, such that

$$
\begin{aligned}
& y_{n}:=\pi\left(x_{n}, \tau_{n}\right) \in H(E, \delta) \\
& z_{n}:=\pi\left(x_{n}, t_{n}\right)=\pi\left(y_{n}, t_{n}-\tau_{n}\right) \in H\left(E, \varepsilon_{n}\right)
\end{aligned}
$$

and

$$
y_{n} \in N, \quad z_{n} \in N
$$

Since $N$ is compact, we can choose two convergent subsequences $\left\{y_{n_{k}}\right\}$ and $\left\{z_{n_{k}}\right\}$. Denote

$$
y=\lim _{k \rightarrow \infty} y_{n_{k}}, \quad z=\lim _{n \rightarrow \infty} z_{n_{k}}
$$

Then $y \in H(E, \delta), z \in E$, and $z \in D^{+}(y)$. So $D^{+}(y) \cap E \neq \varnothing$. By Theorem 3.2, for any $0<\delta_{0}<\delta$, we have $y_{0} \in H\left(E, \delta_{0}\right)$ such that $A^{+}\left(y_{0}\right) \subset E$, which is a contradiction to our assumption, and then the proof is completed.

Remark 5. If $X / E$ is a positively invariant set and $E$ is closed, then Theorem 3.7 holds. To see this is true, one is required only to use Theorem 3.2 and the same discussions such as those in the proof of Theorem 3.7.

Analogous to Theorem 3.3, we can prove the following.

Theorem 3.8. Let $E$ be a closed, positively invariant subset of $X$ with nonempty $E^{2}$ and $\partial E$. Suppose there exists $\alpha>0$ such that $F$ is point dissipative on $S[\partial E, \alpha] \cap E$. Then one of the following statements holds.

(i) The boundary $\partial E$ is not isolated.

(ii) There exists $y \in E^{\circ}$ such that $A^{+}(y) \subset \partial E$.

(iii) There exists $\varepsilon>0$ such that for any $x \in E, \lim _{t \rightarrow \infty} d(\pi(x, t), \partial E) \geqslant \varepsilon$.

The above result gives a description of the flow near the boundary of a closed positively invariant subset $E$ of $X$. It is used extensively in the next section when we discuss persistence, an important concept in population dynamics. 


\section{PERSISTENCE}

Let $X$ be a metric space with metric $d$, and let $F$ be a continuous flow defined on $X$. Let $E$ be a closed subset of $X$ with $\partial E$ and $E^{2}$ nonempty. Throughout this section we suppose that $\mathscr{F}$ is positively invariant over $E$. Then $E^{2}$ is also positively invariant, but the boundary $\partial E$ may not be positively invariant.

Definition 4.1. The flow $\mathscr{F}$ is called

(i) weakly persistent if for all $x \in E$,

$$
\lim _{t \rightarrow \infty} \sup d(\pi(x, t), \partial E)>0
$$

(ii) persistent if for all $x \in E$,

$$
\liminf _{t \rightarrow \infty} d(\pi(x, t), \partial E)>0
$$

(iii) weakly uniformly persistent if there exists $\varepsilon_{0}>0$ such that for all $x \in E$,

$$
\lim \sup d(\pi(x, t), \partial E)>\varepsilon_{0}
$$

(iv) uniformly persistent if there exists $\varepsilon_{0}>0$ such that for all $x \in E$,

$$
\liminf _{t \rightarrow \infty} d(\pi(x, t), \partial E)>\varepsilon_{0}
$$

Note that if the closed set $E$ is invariant, the above definitions of weak persistence, persistence, and uniform persistence coincide with those given by Butler et al. (1986) and Butler and Waltman (1986). Weak uniform persistence was firstly defined by Freedman and Moson (1990). They also discussed the interrelations of these definitions.

Theorem 4.2. Let $X$ be a metric space with metric $d$, $F$ be a continuous flow defined on $X$, and $E \subset X$ be a closed, positively invariant set. Suppose that there exists a constant $\alpha>0$ such that $\mathscr{F}$ is point dissipative on $S[\partial E, \alpha] \cap E$. Then the following statements are equivalent.

(i) is uniformly persistent.

(ii) $F$ is weakly persistent and $\partial E$ is isolated in $E$.

(iii) $\mathscr{F}$ is weakly uniformly persistent.

(iv) There exists $0<\varepsilon_{2}<\alpha$ such that for any $x \in S\left[\partial E, \varepsilon_{2}\right] \cap E$, there exists $T_{x}>0$ such that $\pi\left(x, T_{x}\right) \notin S\left[\partial E, \varepsilon_{2}\right]$. 
(v) There exists $0<\varepsilon_{3}<\alpha$ and a continuous function $P_{1}: S[\partial E, \alpha] \rightarrow R^{+}$satisfying the following conditions:

(a) $P_{1}(x)=0$ iff $x \in \partial E$, and

(b) for any $x \in S\left[\partial E, \varepsilon_{3}\right] \cap E$, there exists $T_{x}>0$ such that $P_{1}\left(\pi\left(x, T_{x}\right)\right)>P_{1}(x)$.

Furthermore, let $\Omega=\Lambda^{+}(S[\partial E, \alpha] \cap E)=\left\{\Lambda^{+}(x) \mid x \in S[\partial E, \alpha] \cap E\right\}$ and $M=\Omega \cap \partial E$. If $M$ is a closed invariant set, then the above conclusions are also equivalent to the following statements.

(vi) $M$ is isolated on $E$ and there is no point $x$ in $E$ satisfying $A^{+}(x) \subset M$.

(vii) There exists $0<\varepsilon_{4}<\alpha$ such that for any $x \in S\left[M, \varepsilon_{4}\right] \cap E$, there exists $T_{x}>0$ such that $\pi\left(x, T_{x}\right) \notin S\left[M, \varepsilon_{4}\right]$.

(viii) There exists $0<\varepsilon_{5}<\alpha$ and $a$ continuous function $P_{2}: S[\partial E, \alpha] \rightarrow R^{+}$satisfying

(a) $P_{2}(x)=0$ iff $x \in \partial E$, and

(b) for any $x \in S\left[M, \varepsilon_{5}\right] \cap E$, there exists $T_{x}>0$ such that $P_{2}\left(\pi\left(x, T_{x}\right)\right)>P_{2}(x)$.

Proof. (i) $\Rightarrow$ (ii), (i) $\Rightarrow$ (iii), and (i) $\Rightarrow$ (iv) are obvious; (ii) $\Rightarrow$ (i) can be easily proved by using Theorem 3.8 .

(iii) $\Rightarrow$ (ii). Let $\varepsilon_{1}>0$ be such that for all $x \in E$, lim sup $\operatorname{su}_{t \rightarrow \infty}$ $d(\pi(x, t), \partial E)>\varepsilon$. Take $0<\varepsilon<\min \left\{\varepsilon_{1}, \alpha\right\}$; then for any $x \in S[\partial E, \varepsilon] \cap \mathscr{E}$, $\Lambda^{+}(x) \not \subset S[\partial E, \varepsilon]$. Hence $\partial E$ is isolated in $E^{2}$.

(iv) $\Rightarrow$ (ii). Let $\varepsilon_{2}>0$ be as in (iv). Then for any $x \in S\left[\partial E, \varepsilon_{2}\right] \cap E$, $\Lambda^{+}(x) \not S\left[\partial E, \varepsilon_{2}\right]$, which shows that $\partial E$ is isolated in $E$. Hence for any $x \in S\left[\partial E, \varepsilon_{2}\right] \cap E$, we have $\lim _{\sup _{n \rightarrow \infty}} d(\pi(x, t), \partial E) \geqslant \varepsilon_{2}>0$.

(iv) $\Rightarrow(v)$. Let $\varepsilon_{2}, \varepsilon_{3}$ be as in (iv) and (v) and let $\varepsilon_{2}=\varepsilon_{3}$. Define $P_{1}(x)=d(x, \partial E), x \in S[\partial E, \alpha]$. Then $P_{1}: S[\partial E, \alpha] \rightarrow R^{+}$is continuous and $P_{1}(x)=0$ iff $x \in \partial E$. For any $x \in S\left[\partial E, \varepsilon_{1}\right] \cap E^{2}$, there exists $T_{x}>0$ such that $\pi\left(x, T_{x}\right) \notin S\left[\partial E, \varepsilon_{2}\right]$. Hence $P_{1}\left(\pi\left(x, T_{x}\right)\right)=d\left(\pi\left(x, T_{x}\right), \partial E\right)>\varepsilon_{2} \geqslant P_{1}(x)$.

(v) $\Rightarrow$ (iv). Let $P_{1}(x)$ and $\varepsilon_{3}$ be as in (v). If there exists $x \in S\left[\partial E, \varepsilon_{3}\right] \cap E$ such that $\gamma^{+}(x) \subset S\left[\partial E, \varepsilon_{3}\right]$, then $\overline{\gamma^{+}(x)} \subset S\left[\partial E, \varepsilon_{3}\right]$.

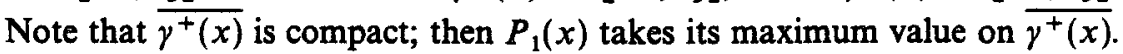
Let $y \in \overline{\gamma^{+}(x)}$ be such that $P_{1}(y) \geqslant P_{1}(z)$ for all $z \in \overline{\gamma^{+}(x)}$. Then $y \in S\left[\partial E, \varepsilon_{3}\right] \cap E$. Since $\gamma^{+}(y) \subset\left\{x: x \in \overline{\gamma^{+}(x)}\right\}$, hence $P_{1}(y) \geqslant P_{1}(z)$ for any $z \in \gamma^{+}(y)$, which contradicts condition (b) in $(v)$.

(ii) $\Rightarrow$ (vi). It is obvious. 
(vi) $\Rightarrow$ (ii). If there exists $x \in E$ such that $\Lambda^{+}(x) \subset \partial E$, then $\Lambda^{+}(x) \subset M$, which contradicts (vi). Hence $\mathscr{F}$ is weakly persistent on $E$. Since $M$ is isolated, there exists $\delta_{0}>0$ such that $S\left[M, \delta_{0}\right]$ is isolated in $E$. If $\partial E$ is not isolated, we can choose a sequence $\left\{\varepsilon_{n}\right\}, 0<\varepsilon_{n}<\min \left\{\delta_{0}, \alpha\right\}$ such that $\lim _{n \rightarrow \infty} \varepsilon_{n}=0$, and a sequence of invariant sets $\left\{W_{n}^{\prime}\right\}, W_{n}^{\prime} \subset$ $S\left[\partial E, \varepsilon_{n}\right], W_{n}^{\prime} \not \subset \partial E$. Define $W_{n}=\overline{W_{n}^{\prime} / M}$, then $W_{n}$ is invariant and $W_{n} \subset N \cap S\left[\partial E, \varepsilon_{n}\right]$, where $N$ is a compact set such that $\left\{\Lambda^{+}(x) \mid x \in\right.$ $S[\partial E, \alpha] \cap E\} \subset N$. Since $S\left[M, \delta_{0}\right]$ is an isolated neighborhood of $M$, then for any $W_{n}$, there exists $y_{n} \in W_{n}$ such that $d\left(y_{n}, M\right)>\delta_{0}$. Let $K$ be the metric space of nonempty compact subsets of $N$ with Hausdorff metric $\rho$; then $K$ is compact (see Whyburn, 1971). Hence we can find a convergent subsequence of $\left\{W_{n}\right\}$, which we also relabel as $\left\{W_{n}\right\}$, such that $\rho\left(W_{n}, W\right) \rightarrow 0$ as $n \rightarrow \infty$, where $W \in K$. Clearly $W \subset \partial E$ and $W$ is invariant; then $W \subset M$. Without loss of generality, we suppose that $\left\{y_{n}\right\}$ is convergent. Let

$$
y=\lim _{n \rightarrow \infty} y_{n}
$$

Then $y \in W$. On the one hand, $d\left(y_{n}, M\right)>\delta_{0}$ implies that $d(y, M) \geqslant \delta_{0}>0$. On the other hand, $y \in W \subset M$ implies $d(y, M)=0$, which is impossible.

$$
\begin{aligned}
& \text { (iv) } \Rightarrow \text { (vii) and (v) } \Rightarrow \text { (viii). Obvious. } \\
& \text { (vii) } \Rightarrow \text { (vi). Similar to the proof of (iv) } \Rightarrow \text { (ii). } \\
& \text { (viii) } \Rightarrow \text { (vi). Similar to the proof of }(v) \Rightarrow \text { (ii). }
\end{aligned}
$$

Remark 6. Theorem 4.2 may be viewed as a unified and generalized theorem combining results of Fonda (1988), Freedman and Moson (1990), Hofbauer (1989), and Hofbauer and So (1989).

We denote the restriction of $\mathscr{F}$ to $\partial E$ by $\partial \mathscr{F}$ and note that $\partial E$ is, in general, not positively invariant. Let $N$ be the maximal invariant set of $\partial \mathscr{F}$ on $\partial E$. Suppose $N$ is a closed invariant set and there exists a cover $\left\{N_{\alpha}\right\}_{\alpha \in A}$ of $N$, where $A$ is a nonempty index set, $N_{\alpha} \subset \partial E, N \subset \bigcup_{\alpha \in A} N_{\alpha}$, and $N_{\alpha}(\alpha \in A)$ are pairwise disjoint closed invariant sets. Furthermore, we propose the following hypothesis.

(H) (a) All $N_{\alpha}$ are isolated invariant sets of the flow

(b) $\left\{N_{\alpha}\right\}_{\alpha \in A}$ is acyclic, that is, any finite subset of $\left\{N_{\alpha}\right\}_{\alpha \in A}$ does not form a cycle (see Butler and Waltman, 1986).

(c) Any compact subset of $\partial E$ contains, at most, finitely many sets of $\left\{N_{\alpha}\right\}_{\alpha \in A}$. 
Theorem 4.3. Let $E$ be a closed positively invariant subset of $X$ on which a continuous flow $\mathscr{F}$ is defined. Suppose there is a constant $\alpha>0$ such that is point dissipative on $S[\partial E, \alpha] \cap E^{2}$ and the assumption (H) holds. Then the flow is uniformly persistent if and only if

$$
W^{+}\left(N_{\alpha}\right) \cap S[\partial E, \alpha] \cap \stackrel{E}{=}=\varnothing
$$

for any $\alpha \in A$, where $W^{+}\left(N_{\alpha}\right)=\left\{y \in X: \Lambda^{+}(y) \subset N_{\alpha}\right\}$.

The proof is similar to that given by Butler et al. (1986) and Butler and Waltman (1986).

Remark 7. Theorem 4.3 generalizes Theorem 3.1 of Butler and Waltman (1986), Theorem 4.2 of Hofbauer and So (1989), Theorem 2 of Garay (1989), and Theorem 3 of Teng and Duan (1990).

\section{EXAMPLES}

In this section we consider some examples to illustrate our results. In a recent paper, Hutson and Schmitt (1992) survey results leading to uniform persistence in ecological systems. All their theorems require dissipativity of the models. On the other hand, the classical Lotka-Volterra models are not necessarily dissipative, but if so, they lead to exhibit persistence which is not uniform. Here we give examples of systems which are either not dissipative or not invariant on the axes, but which are uniformly persistence.

Example 1. In this example we consider a simple model of competition with a source term for one of the competitors, without which it cannot survive. The model is given as follows:

$$
\left\{\begin{array}{l}
\frac{d x_{1}}{d t}=x_{1}\left(1-x_{1}-a x_{2}\right) \\
\frac{d x_{2}}{d t}=x_{2}\left(-1-b x_{1}-x_{2}\right)+\varepsilon
\end{array}\right.
$$

In the case $\varepsilon=0, x_{2} \rightarrow 0$ as $t \rightarrow 0$. However, by our results, if

$$
0<\varepsilon<\frac{1}{4}\left[\left(1+\frac{a}{2}\right)^{2}-1\right]
$$

then system (1) is uniformly persistence. 
Example 2. Here we consider a system of predator-prey type

$$
\left\{\begin{array}{l}
\frac{d x}{d t}=x(1-y(1+x)) \\
\frac{d y}{d t}=y\left(-1+x^{2}-y\right)
\end{array}\right.
$$

When $y=0$, the subsystem $d x / d t=x$ is clearly not dissipative. However, our results show that the system is persistence with a locally stable interior equilibrium.

As pointed out by Thieme (1993), persistence theory has so far focused rather on ecological models than epidemiological models. In epidemiology, the question of persistence can be posed in a twofold way: persistence of the host population, i.e., the disease does not extinguish the host; and persistence (or endemicity) of the disease, i.e., the disease does not go extinct itself. In the following we consider a SEIRS epidemiological model following Liu et al. (1987) and Hethcote and van den Driessche (1991).

Example 3. The host population is subdivided into susceptible $(S)$, exposed $(E)$, infections $(I)$, and recovered $(R)$ individuals:

$$
\left\{\begin{array}{l}
\frac{d S}{d t}=-\lambda I^{p} S^{q}+\mu-\mu S+\delta R \\
\frac{d E}{d t}=\lambda I^{p} S^{q}-(\varepsilon+\mu) E \\
\frac{d I}{d t}=\varepsilon E-(r+\mu) I \\
\frac{d R}{d t}=r I-(\delta+\mu) R \\
S+E+I+R=1
\end{array}\right.
$$

All parameters are assumed to be positive. By using the assumption $S+E+I+R=1$, the model can be reduced to the following three-dimensional system

$$
\left\{\begin{array}{l}
\frac{d E}{d t}=\lambda I^{p}(1-E-I-R)^{q}-(\varepsilon+\mu) E \\
\frac{d I}{d t}=\varepsilon E-(r+\mu) I \\
\frac{d R}{d t}=r I-(\delta+\mu) R
\end{array}\right.
$$


We can see that the region $B=\{(E, I, R): E \geqslant 0, I \geqslant 0, R \geqslant 0$, $E+I+R \leqslant 1\}$ is positively invariant with respect to the model. There always exists a unique equilibrium $(0,0,0)$ on the boundary of $B$ which corresponds to the disease-free state. By Theorem 4.3, we can see that uniform persistence of system (4) is equivalent to instability of the diseasefree equilibrium $(0,0,0)$. Combine the local stability analysis for this trivial equilibrium by Liu et al. (1987) and Theorem 4.3; we know that system (4) and hence system (3) are uniformly persistence if and only if $0<p<1$ or $p=1, \delta>1$.

\section{ACKNOWLEDGMENTS}

The authors would like to thank Professor G. Sell and the referee for helpful comments and suggestions on the original version of this paper. H.I.F.'s research was partially supported by the Natural Sciences and Engineering Research Council of Canada, Grant NSERC A4823. This research was carried out while S.R. was a postdoctoral fellow at the Fields Institute for Research in Mathematical Sciences and was partially supported by the Ministry of College and University of Ontario and the Natural Sciences and Engineering Research Council of Canada.

\section{REFERENCES}

Bhatia, N. P. (1969). Dynamical systems. In Kuhn, H. W., and Szegö, G. P. (eds.), Mathematical Systems Theory and Economics, Springer-Verlag, New York, pp. 1-10.

Bhatia, N. P. (1970). Attraction and non-saddle sets in dynamical systems. J. Diff. Eqs. 8, 229-249.

Bhatia, N. P., and Szegö, G. P. (1970). Stability Theory of Dynamical Systems, SpringerVerlag, Berlin.

Butler, G. J., and Waltman, P. (1986). Persistence in dynamical systems. J. Diff. Eqs. 63, 255-263.

Butler, G. J., Freedman, H. I., and Waltman, P. (1986). Uniformly persistent systems. Proc. Am. Math. Soc. 96, 425-430.

Conley, C. (1978). Isolated Invariant Sets and the Morse Index, CBMS, Vol. 38, Providence, RI.

Dunbar, S. R., Rybakowski, K. P., and Schmitt, K. (1986). Persistence in models of predatorprey populations with diffusion. J. Diff. Eqs. 65, 117-138.

Fernandes, M., and Zanolin, F. (1990). Repelling conditions for boundary sets using Liapunov-like functions. II. Persistence and periodic solutions. J. Diff. Eqs. 86, 33-58.

Fonda, A. (1988). Uniformly persistent semidynamical systems. Proc. Am. Math. Soc. 104, 111-116.

Freedman, H. I., and Moson, P. (1990). Persistence definitions and their connections. Proc. Am. Math. Soc. 109, 1025-1033.

Freedman, H. I., and Ruan, S. (1994). Uniform persistence in functional differential equations. J. Diff. Eqs. (in press). 
Freedman, H. I., and So, J. (1989). Persistence in discrete semidynamical systems. SLAM J. Math. Anal. 20, 930-938.

Freedman, H. I., and Waltman, P. (1984). Persistence in models of three interacting predatorprey populations. Math. Biosci. 68, 213-231.

Garay, B. M. (1989). Uniform persistence and chain recurrence. J. Math. Anal. Appl. 139, 372-381.

Gard, T. C. (1987). Uniform persistence in multispecies population models. Math. Biosci. 85, 93-104.

Gard, T. C., and Hallam, T. G. (1979). Persistence of food webs. I. Lotka-Volterra food chains. Bull. Math. Biol. 41, 877-891.

Hale, J. K. (1988). Asymptotic Behavior of Dissipative Systems, Am. Math. Soc., Providence, RI.

Hale, J. K., and Waltman, P. (1989). Persistence in infinite dimensional systems. SIAM J. Math. Anal. 20, 388-395.

Hethcote, H. W., and van den Driessche, P. (1991). Some epidemiological models with nonlinear incidence. J. Math. Biol. 29, 271-287.

Hofbauer, J. (1981). A general cooperation theorem for hypercycles. Monatsh. Math. 91, 233-240.

Hofbauer, J. (1989). A unified approach to persistence. Acta Appl. Math. 14, 11-22.

Holbauer, J., and Sigmund, K. (1988). Dynamical Systems and the Theory of Evolution, Cambridge University Press, Cambridge.

Hofbauer, J., and So, J. (1989). Uniform persistence and repellers for maps. Proc. Am. Math. Soc. 107, 1137-1142.

Hutson, V. (1984). A theorem on average Liapunov functions. Monalsh. Math. 98, 267-275.

Hutson, V., and Schmitt, K. (1992). Permanence and the dynamics of biological systems. Math. Biosci. 111, 1-71.

Liu, W. M., Hethcote, H. W., and Levin, S. A. (1987). Dynamical behavior of epidemiological models with nonlinear incidence rates. J. Math. Biol. 25, 359-380.

Sell, G. R. (1967). Nonautonomous differential equations and topological dynamics. I. The basic theory. Trans. Am. Math. Soc. 127, 247-262.

Sell, G. R., and Sibuya, Y. (1967). Behavior of solutions near a critical point. In Harris, W. A., Jr., and Sibuya, Y. (eds.), Proceedings United States-Japan Seminar on Differential and Functional Equations, Benjamin, New York, pp. 501-506.

Tang. M. (1990). Persistence in a higher dimensional population dynamical systems. Acta Math. Appl. Sinica 13, $431^{\star}-443$.

Teng, Z.-D., and Duan, K.-C. (1990). Persistence in dynamical systems. Q. Appl. Math. 48, $463-472$.

Thieme, H. R. (1993). Persistence under relaxed point-dissipativity (with application to an endemic model). SIAM J. Math. Anal. 24, 407-435.

Ura, T., and Kimura, I. (1960). Sur le courant extérieur à une région invariante: Théorème de Bendixson. Comm. Math. Univ. St. Paul 8, 23-39.

Waltman, P. (1992). A brief survey of persistence in dynamical systems. In Busenberg, S., and Marteli, M. (eds.), Delay Differential Equations and Dynamical Systems, Springer-Verlag, New York, pp. 31-40.

Whyburn, G. T. (1942). Analytic Topology, Am. Math. Soc. Colloq. Publ. Vol. 28, Providence, RI.

Yang, F., and Ruan, S. (1992). A generalization of the Butler-McGehee lemma and its applications in persistence theory. Preprint. 